\title{
Analysis of Internal Structure of Cement-Stabilized Macadam Based on Industrial CT Scanning
}

\author{
Chunyu Liang, ${ }^{1}$ Yinghan Wang, ${ }^{1}$ Guojin Tan $\left(\mathbb{D},{ }^{1}\right.$ Lidong Zhang, ${ }^{2}$ Yutong Zhang, \\ and Zhihua $\mathrm{Yu}^{3}$ \\ ${ }^{1}$ College of Transportation, Jilin University, Changchun 130025, China \\ ${ }^{2}$ Jilin Provincial Bureau of High-grade Highway Construction, Changchun 130000, China \\ ${ }^{3}$ CCCC Second Highway Engineering Co. Ltd., Xi'an 710065, China
}

Correspondence should be addressed to Guojin Tan; tgj@jlu.edu.cn

Received 28 May 2020; Revised 15 July 2020; Accepted 3 August 2020; Published 27 August 2020

Academic Editor: Zhanping You

Copyright $(92020$ Chunyu Liang et al. This is an open access article distributed under the Creative Commons Attribution License, which permits unrestricted use, distribution, and reproduction in any medium, provided the original work is properly cited.

The composition and structure of cement-stabilized macadam (CSM) is an important factor affecting its performance. Aiming at the typical suspension dense structure and skeleton dense structure, the static pressure and vibration methods were used to prepare the CSM samples. The three-dimensional internal structure characteristics of CSM were analyzed and studied by industrial CT scanning technology. By using digital image and 3D reconstruction technology, the structural parameters such as the number of 3D voids, the gradation of voids, the distribution of voids along the height direction, and the coefficient of variation of the proportion of coarse aggregate area were obtained. The results show that more than $80 \%$ of the voids in CSM are less than $1 \mathrm{~mm}^{3}$, and the number of large voids of skeleton dense gradation is more than that of suspension dense gradation under the two compaction methods. The distribution of internal voids along the height of CSM specimens cannot be changed by the compaction mode, but the vibration method can reduce its void ratio. Under different compaction methods, the variation coefficient of the coarse aggregate area ratio of two kinds of CSM mixture generally has the same change trend, and the vibration method can make its inner structure more uniform.

\section{Introduction}

The cement-stabilized macadam (CSM) is a kind of mixture compacted of graded macadam, water, and cement according to the principle of density. The performance of CSM with different composition structures is quite different, and gradation and compaction method are the important factors that directly affect its composition structure. The selection of the compaction method of specimens has always been one of the important contents in the mix proportion design of CSM. The internal structure of CSM include the size and number of voids, void distribution, coarse aggregate structural uniformity, and filling degree of fine aggregate. Because it is difficult to obtain the internal structural of CSM directly, it has been impossible for effective analysis and research. The emergence of industrial Computed Tomography $(\mathrm{CT})$ provides a convenient and reliable way to solve this problem. Industrial CT technologies can present the internal structure of the detected sample clearly and visually in the form of two-dimensional or three-dimensional image without damaging the detected sample and is praised as the best nondestructive testing and evaluation technology [1]. Using industrial CT scanning, the internal composition structure of CSM mixture can be obtained intuitively, and its structural characteristics can be characterized by the corresponding parameter indexes.

At present, using industrial CT technology to study the microstructure of civil engineering materials mainly focuses on asphalt mixture, cement concrete, and rock. CT scanning of asphalt mixture involves the study of its internal composition structure, aggregate profile, and uniformity. Some researchers used industrial CT technology to identify the internal structure of asphalt concrete and established the evaluation method of asphalt mixture uniformity $[2,3]$. 
Hunter et al. adopted digital image processing (DIP) technology to evaluate the uniformity of asphalt mixture specimens with different compaction methods [4]. In order to evaluate the compaction uniformity in the dynamic compaction process of asphalt mixture, X-ray computed tomography technology was introduced to scan the specimens under various rotating compaction times, and then their longitudinal pore structure distribution was calculated and compared, respectively [5]. CT scanning images and standard normal distribution parameters were also used to evaluate the homogeneous of asphalt mixture and the geometric properties of the aggregate distribution [6]. Using industrial CT to obtain the internal structure of asphalt concrete specimen, the two-dimensional contour of particles in the image was extracted by the digital image processing method. The reasonable sampling interval angle was determined combining with the concept of equivalent diameter, and the value of Fourier transform order was qualitatively analyzed [7].

CT scanning and image processing techniques can also be used to analyze the size, number, and distribution of voids in composites. For example, previous study extracted the information of voids distribution in rotating compacted asphalt mixture specimens by CT techniques, analyzed the voids distribution in vertical and horizontal directions, and showed the influence of voids through mechanical property tests [8]. Using industrial CT, the void distribution of asphalt mixture with different compaction methods under the same gradation were analyzed and studied [9]. Scholars scanned the Marshall specimen of the drainage asphalt mixture and adopted the digital image technology to obtain the internal structure composition of the drainage asphalt mixture. Based on these works, the voidage data were quantitatively analyzed after the section image processing and the connected void path in the drainage asphalt mixture was tracked [10]. Wu et al. also studied the surface porosity along depth of asphalt concrete compacted by different methods and actual pavement coring specimens with different layers [11].

For the study of concrete and rock, industrial CT is mostly used to observe and analyze the changing law and the damage of the internal structure of the material under force or freeze-thawing conditioning, etc. The representative research includes using industrial CT technology to study the variation law of microscopic pore characteristics of concrete material under different freeze-thaw cycles [12]. Gao et al. used industrial CT technology to study the microscopic crack propagation law of lightweight aggregate concrete under the condition of loading and nonloading [13]. Based on the research of industrial CT technology, Mao et al. established the 3D model of concrete, analyzed the feasibility of the model with finite element software, and provided the modeling method to study the microscopic properties of concrete materials by the numerical method [14]. The changing trend of porosity before and after loading of coal petrography was studied by industrial CT technology, and the density distribution image of its CT section and dynamic damage process was analyzed [15].

To sum up, though industrial CT has been widely used in many fields, there are not enough studies on the internal structure of CSM materials. It is of great significance to study the internal structural characteristics of the CSM mixture under different laboratory-fabricated methods for its structural composition design and compaction control. In this paper, the internal structure of CSM was studied by industrial CT scanning, and the influence of different compaction methods on the internal structure void characteristics and coarse aggregate distribution uniformity was studied. The results would provide reference and theoretical basis for the mix proportion design of CSM, selection of specimen fabricated method, and compaction process and control during construction.

\section{Preparation of CSM Specimen}

In this study, the $15 \mathrm{~cm} \times 15 \mathrm{~cm}$ cylindrical CSM specimen was used. Combined with engineering application, the cement was P.O.42.5 and the type of aggregate was basalt. According to the gradation range in the Technical Guidelines for Construction of Highway Road-bases [16] and the existing research results of the research group, two kinds of cement-stabilized macadam gradation forms, skeleton dense structure and suspended dense structure, were selected and expressed by GJ and XF. The passing rate of each sieve pore of the two-design gradation is shown in Table 1.

Because this study mainly focuses on the influence of gradation and compaction method on the internal structure of CSM, the cement dosage of the test specimen was $5 \%$ and the compaction degree was $98 \%$ of the maximum dry density. The specimens were made by static compaction (JY) and vibration compaction method (ZD), respectively, based on the Test Methods of Materials Stabilized with Inorganic Binders for Highway Engineering [17]. The optimum moisture content and maximum dry density of the compaction test were shown in Table 2, in which the vibration time of vibration compaction was $120 \mathrm{~s}$. After curing at the standard condition for 28 days, the test sample was drilled by core drilling machine, then cutting its both ends to obtain the scanning specimen of $10 \mathrm{~cm} \times 10 \mathrm{~cm}$ CSM.

\section{Test and Analysis Method}

3.1. CT Scanning Principle and Method. The basic working principle of industrial CT system is that when the X-ray emitted by the light source penetrates the test object, the test object will absorb some photons in the X-ray, which will cause the X-ray energy to decay. After penetrating through the test object, the remaining unabsorbed photons are projected onto the detector panel. The energy intensity of the $\mathrm{X}$-ray received by the detector is converted into an electrical signal through a converter, which is then sent into the computer to carry out the digital processing to show the scanning section in the form of image, as shown in Figure 1.

When the test material rotates for one cycle and the scanning is finished, the detector receives the X-ray absorption data from different positions of the test material and obtains the cross-sectional image of the test material after computer calculation and processing. Compared with the conventional experimental research methods, industrial 
TABLE 1: Gradation design table of CSM.

\begin{tabular}{lcc}
\hline Mesh size $(\mathrm{mm})$ & \multicolumn{2}{c}{ Percentage of passing each sieve pore (\%) } \\
Skeleton dense structure & $\begin{array}{c}\text { Suspended dense } \\
\text { structure }\end{array}$ \\
\hline 31.5 & 100 & 100 \\
26.5 & 96.8 & 100 \\
19.0 & 77.4 & 82.9 \\
9.5 & 48.0 & 53.0 \\
4.75 & 30.9 & 36.2 \\
2.36 & 19.0 & 22.0 \\
0.6 & 10.0 & 10.6 \\
0.075 & 1.5 & 2.1 \\
\hline
\end{tabular}

CT scanning can provide the sectional images containing the information such as the geometric structure and void structure of the specimen. It has the advantages of intuitive imaging, high resolution, and not limited by the structure of the specimen.

The scan of CSM samples used the industrial CT scanner produced by General Company, as shown in Figure 2. The rated voltage and the rated current were $180 \mathrm{~V}$ and $110 \mu \mathrm{A}$, and the scanning mode was every 0.36 degrees once. When the core sample of the specimen was scanned along the height direction, 1000 scanning images can be obtained from a core sample, as shown in Figure 3, and the picture size was 1000 pixels $\times 1000$ pixels.

3.2. 3D Void Calculation. Void refers to the pores in the CSM specimen, which is the unfilled space inside the specimen. The number and size of voids are closely related to the performance of the CSM. Carrying out CT scanning on the specimens prepared above to obtain the cross-section of the internal structure, the relevant structural parameters of the CSM could be analyzed using the picture processing software.

Referring to the related research of the asphalt mixture [18], the image of CSM samples obtained by industrial CT scanning was imported into VGStudio MAX 2.2 software for $3 \mathrm{D}$ reconstruction. The test parameters were set, and the matching defect analysis module was selected to accurately calculate the $3 \mathrm{D}$ void volume and void quantity of the specimen. The maximum void volume to be measured was set to $600 \mathrm{~mm}^{3}$ and the minimum void volume was set to $0 \mathrm{~mm}^{3}$ by the "defect extension" option.

The $3 \mathrm{D}$ reconstruction of the CSM specimen and its voids extracted by the software were shown in Figure 4, and the resulting void information includes the spatial $3 \mathrm{D}$ coordinates of each void inside the specimen, void volume, void surface area, and void ratio.

3.3. Digital Image Processing. The purpose of CSM mixture compaction is to make the coarse aggregate close to form a skeleton under the action of load, and the fine aggregate can be well filled in it, but the arrangement of aggregate particles cannot be judged directly and accurately in the compaction process. With the emergence and development of image technology, it is possible to obtain the internal information of the target by scanning and photographing. Through the image processing and analysis, the mesostructure of the mixture was obtained.

At present, the application of image technology in the asphalt mixture is more in-depth because there is a clear boundary between aggregate and asphalt in asphalt concrete, which is convenient for information processing and extraction of mesostructure, while it is less used in the CSM mixture. The CSM mixture is composed of graded macadam and cement mortar, and there is no distinct mortar layer such as cement concrete. Furthermore, in the continuous graded gravel, each particle size exists, which makes it difficult to extract the edge of coarse aggregate. Through industrial CT scanning, Image-Pro Plus 6.0 and Matlab software were used to process the scanning image; then, the approximate distribution of coarse aggregate in CSM was extracted. Figure 5 was the binary image of the treated CSM coarse aggregate.

As can be seen from Figure 5, the coarse aggregate particles show no obvious profile because the essence of industrial CT scanning is to obtain the internal information of the scanned specimens by computer according to the weakening and absorption characteristics of radiation in the tested objects, while each part of the CSM has similar properties and the adhesion effect between aggregates is serious, so we can only extract the similar coarse aggregate information.

In the CSM mixture, the coarse aggregate forms the main skeleton under the action of extrusion force because of its large volume and high strength, and the fine aggregate plays the role of filling skeleton, so the composition structure of the cement-stabilized macadam is largely dependent on the distribution of the coarse aggregate. Therefore, the distribution state of coarse aggregate has an important influence on the internal structure of CSM mixture. The composition of each part of CSM mixture is very similar, so it is impossible to extract the coarse aggregate shape directly. In this paper, referring to the research thoughts of $\mathrm{Wu}$ et al. [19], the section picture of CSM was divided into 12 sector regions by software, and the coarse aggregate in the sector area was extracted; then, the area ratio parameters of coarse aggregate were obtained, as shown in Figure 6.

The proportion of the coarse aggregate area refers to the ratio of the coarse aggregate area to sectorial area of section picture. The extracted sector shape was shown in Figure 7; then, the structure distribution state of the CSM coarse aggregate was analyzed by this parameter.

\section{Results and Discussion}

\subsection{Analysis of Void Characteristics of CSM}

4.1.1. Void Gradation. Void gradation refers to the percentage of pores counted in each volume to the total number of pores in CSM specimens. Statistical analysis was carried out on the void parameters calculated by VGS software to obtain the void gradation of CSM specimens of different 
TABLE 2: Sample properties.

\begin{tabular}{|c|c|c|c|c|}
\hline Sample ID & Compaction method & Gradation & $\begin{array}{l}\text { Maximum dry density } \\
\left(\mathrm{g} / \mathrm{cm}^{3}\right)\end{array}$ & $\begin{array}{c}\text { Optimum moisture content } \\
(\%)\end{array}$ \\
\hline GJZD & \multirow{2}{*}{ Vibration compaction } & Skeleton dense structure & 2.384 & 4.7 \\
\hline XFZD & & Suspended dense structure & 2.374 & 4.9 \\
\hline GJJY & \multirow{2}{*}{ Static compaction } & Skeleton dense structure & 2.373 & 4.8 \\
\hline XFJY & & Suspended dense structure & 2.362 & 5.1 \\
\hline
\end{tabular}

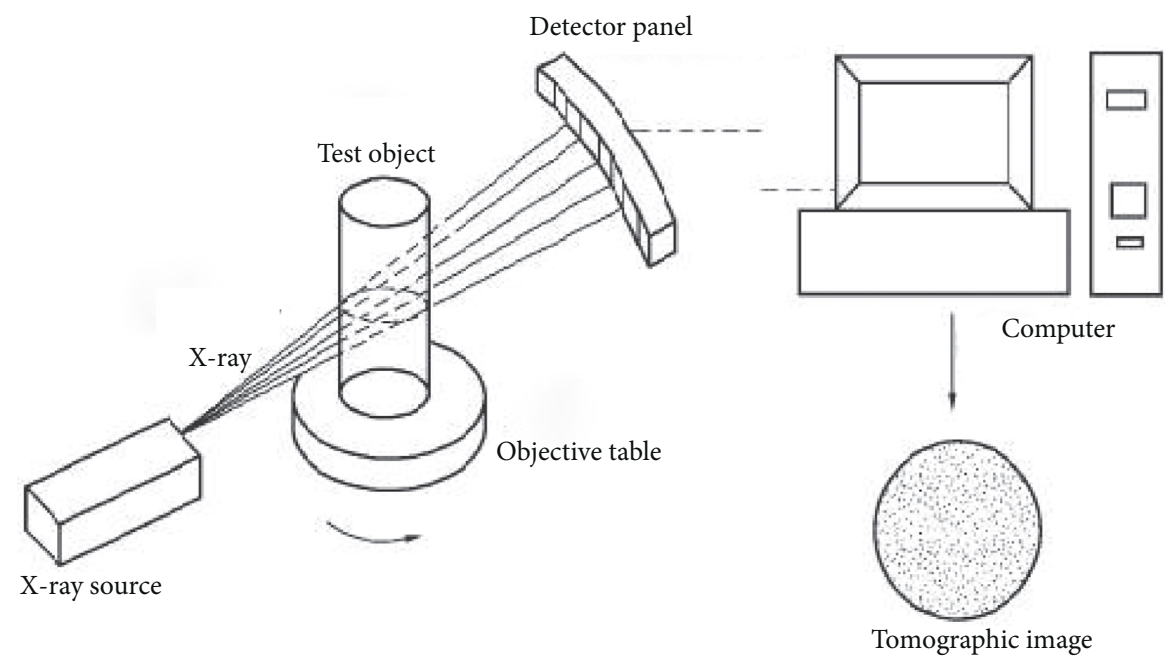

FIGURE 1: CT imaging schematic diagram.

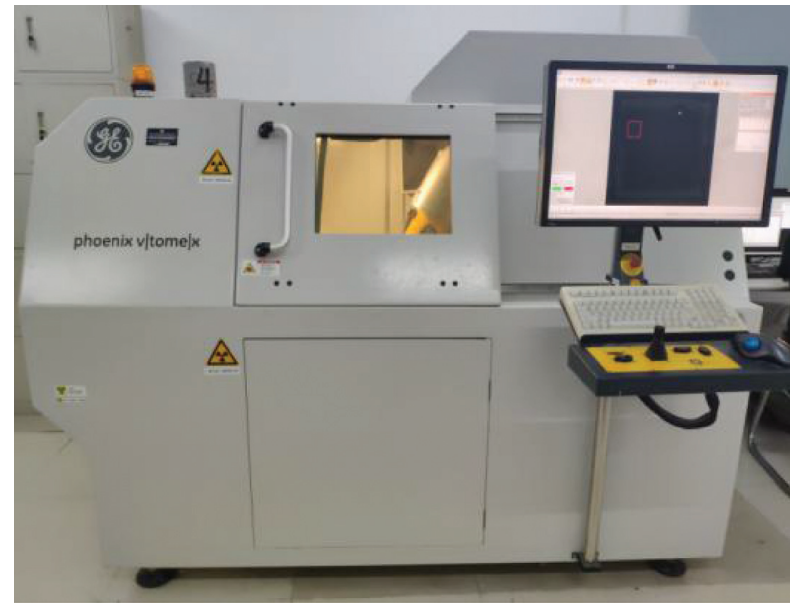

Figure 2: Industrial CT scanning test device.

grading under different compaction methods. The results were shown in Figure 8.

Figure 8 shows that more than $80 \%$ of the voids in CSM are voids with volume less than $1 \mathrm{~mm}^{3}$. On the whole, the number of large volume voids in skeleton dense gradation, especially $0.1 \sim 0.5 \mathrm{~mm}^{3}$, is more than that in suspension dense gradation.

Under static compaction, the void volume between suspension gradation and skeleton gradation is quite different. About $50 \%$ of the internal voids of suspended dense gradation are less than $0.1 \mathrm{~mm}^{3}$, while the most internal voids of skeleton dense gradation are between $0.1 \mathrm{~mm}^{3}$ to

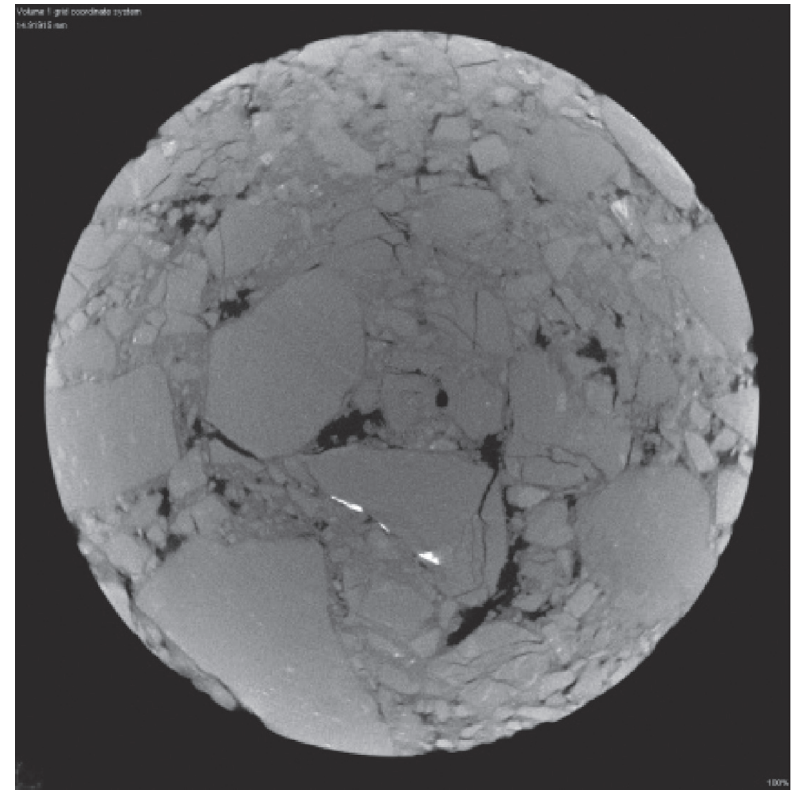

Figure 3: CT scanning image of CSM specimen.

$0.5 \mathrm{~mm}^{3}$. This is maybe because there are few coarse aggregate and relatively more fine aggregate in the suspension dense gradation, and the static compaction method will further lead to the partial coarse aggregate breaking, and the proportion of its voids less than $1 \mathrm{~mm}^{3}$ increase.

Under the vibration mode, the internal void volume of the two kinds of gradation are most in the range of 

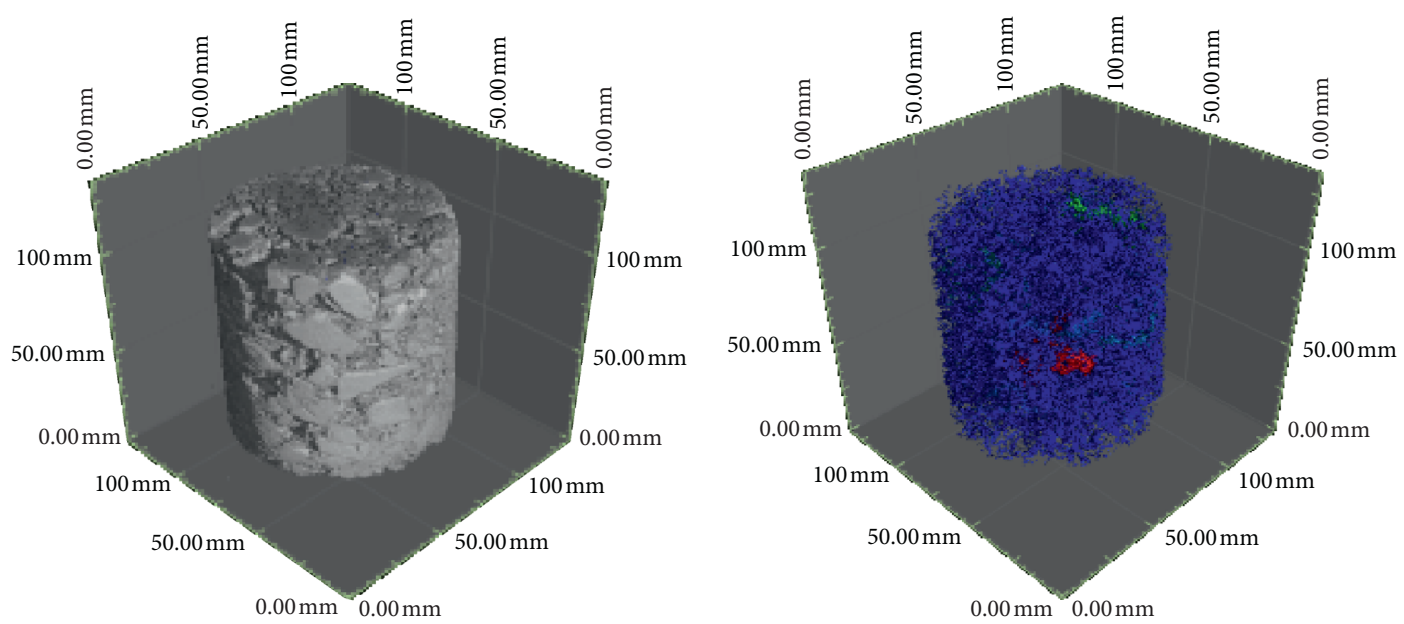

FIgURE 4: Three-dimensional void of CSM.

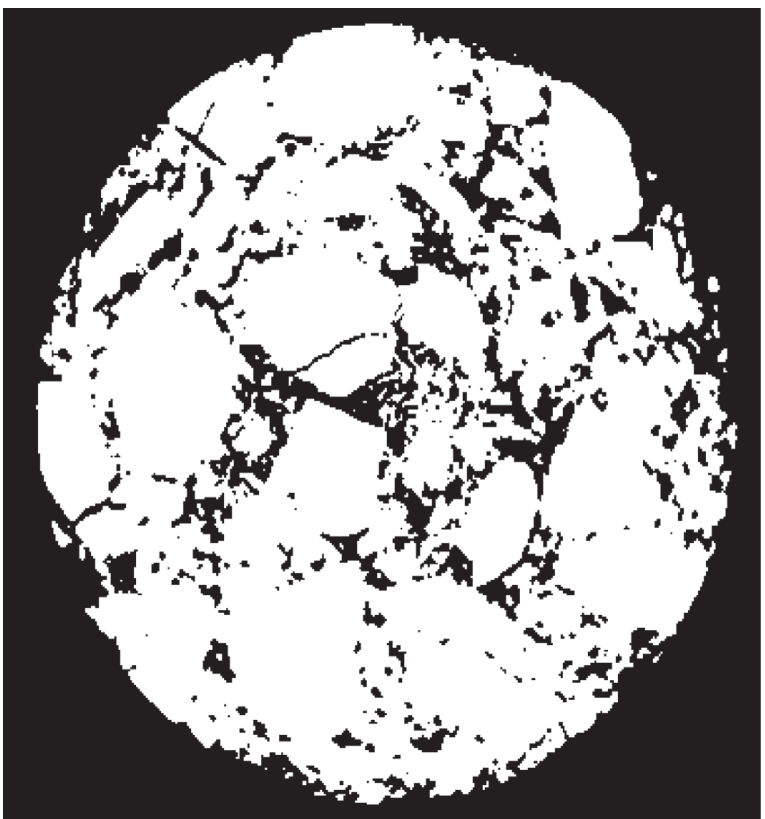

FIGURE 5: Binary picture of coarse aggregate.

$0.1 \mathrm{~mm}^{3} \sim 0.5 \mathrm{~mm}^{3}$, but it is obvious that the skeleton dense gradation has more large void, which is about $10 \%$ more than that of suspension dense gradation.

In general, the void volume of skeleton dense gradation is larger than that of suspension dense gradation under the two compaction methods.

4.1.2. Characteristics of Void Plane Distribution. The voidage gradation obtained by VGS software only obtains the volume size and quantity distribution of the internal voids in CSM, and it cannot reflect the specific distribution of voids along the height. To solve this problem, gray scale processing of continuous section image of CSM scanned by CT was carried out using matlab software. Figure 9 was the processed gray image. Then, the contrast, brightness, and

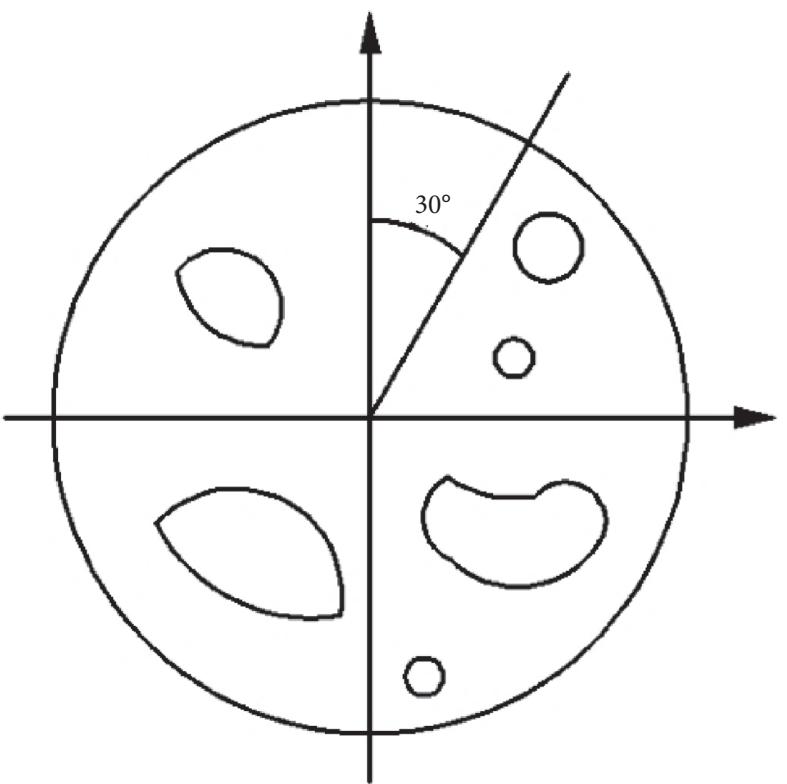

FIGURE 6: Sector area division.

gamma value were adjusted by Image-Pro Plus software to process these gray images. Through such methods the voids' information of the internal structure of CSM could be obtained, and the distribution of voids with the height of the specimen could also be obtained accurately. Figure 10 showed a picture after void extracted by Image-Pro Plus software.

Because of the damage caused by the coring process of the specimen preparation, the CT picture information of the specimen on the top and bottom surfaces may not be complete. Therefore, the incomplete parts with $0.5 \mathrm{~cm}$ height on the upper and lower surfaces were discarded in the analysis, and the continuous cross-section pictures in the $9 \mathrm{~cm}$ middle height were taken to study.

The surface voidage is defined as the ratio of the void area to cross-section area in the tomographic image. The 


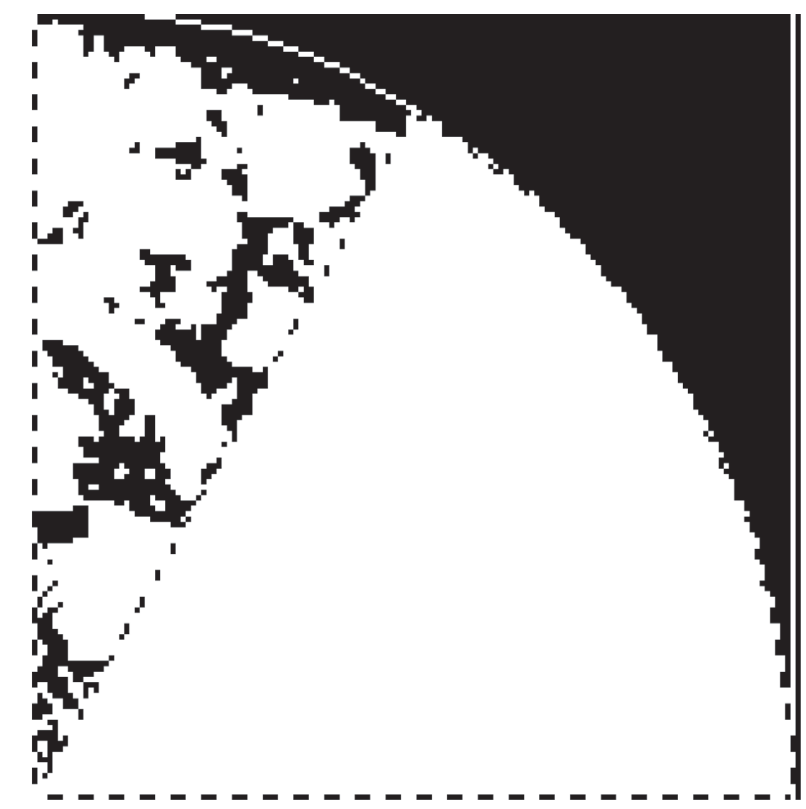

FIgURE 7: Extraction sector diagram of coarse aggregate.

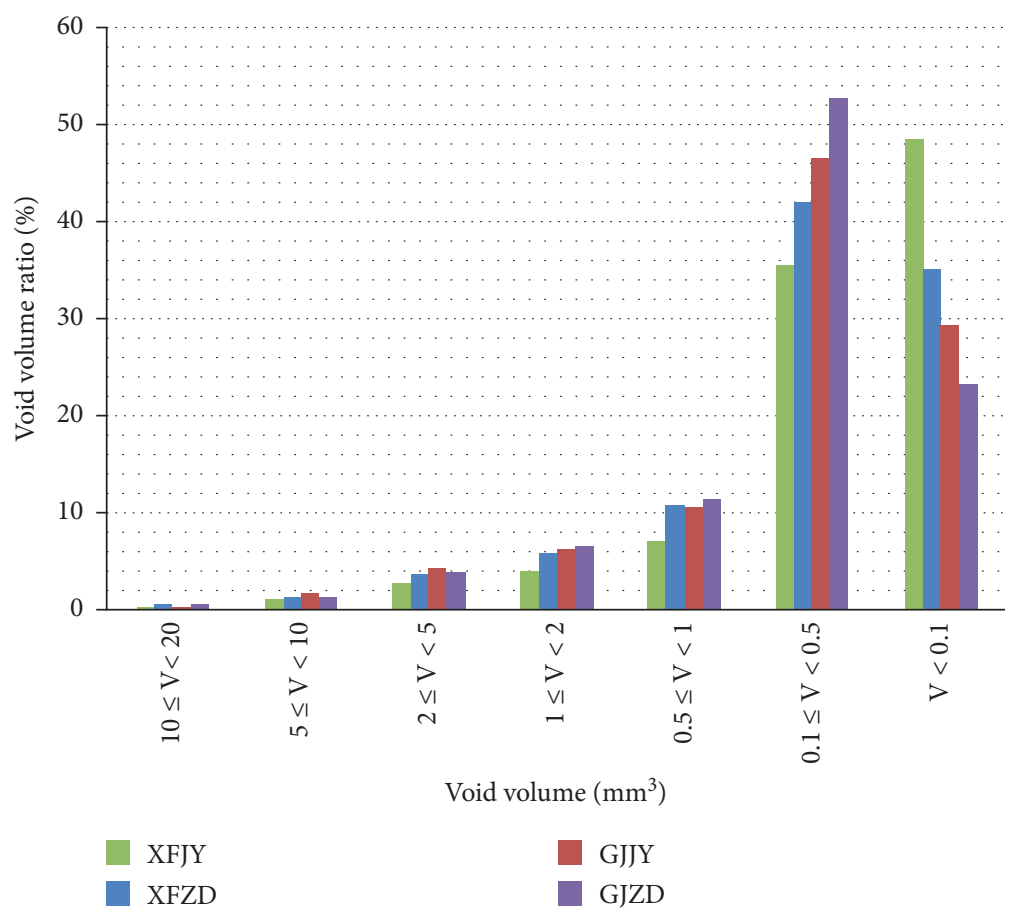

FIgURE 8: Void gradation distribution of CSM.

distribution of the surface voidage of CSM was statistically analyzed by Image-Pro Plus software, and the results were shown in Table 3.

From Table 3, it can be seen that the maximum and minimum surface voidage of two kinds of CSM is quite different, which indicates that the void distribution is uneven and fluctuates obviously. The average surface voidage of suspension dense gradation is higher than that of skeleton dense gradation, and the void distribution of skeleton dense gradation is more uniform. Under the vibration compaction mode, the surface voidage of the two gradations is reduced, which means that the void distribution of CSM can be more uniform when the specimen is fabricated by the vibration method. From the point of view of coefficient of variation, the minimum coefficient of variation is obtained in the skeleton gradation under static compaction, and the 


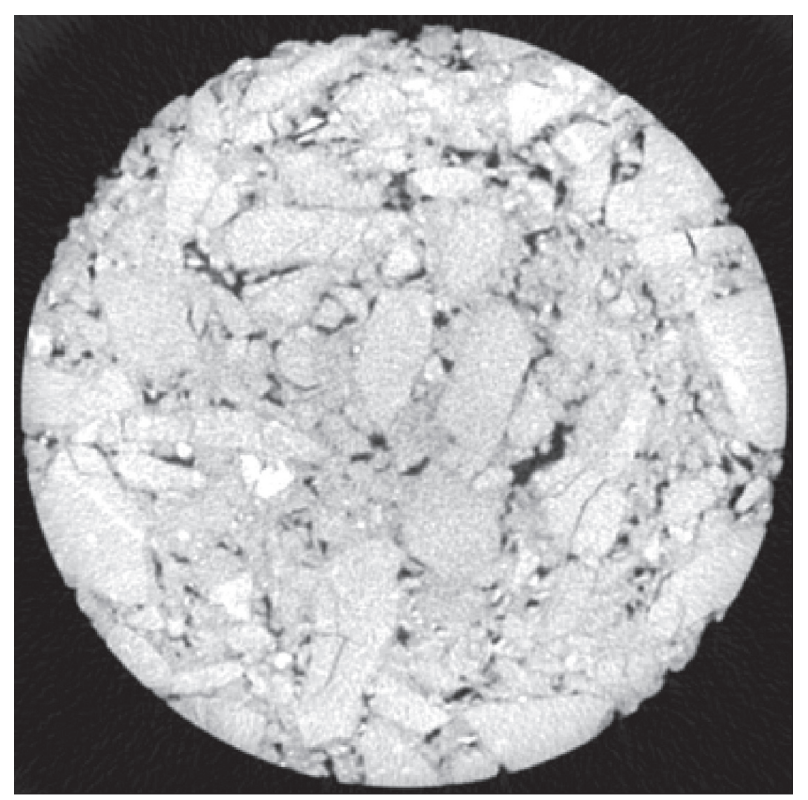

FIgURE 9: The processed gray image.

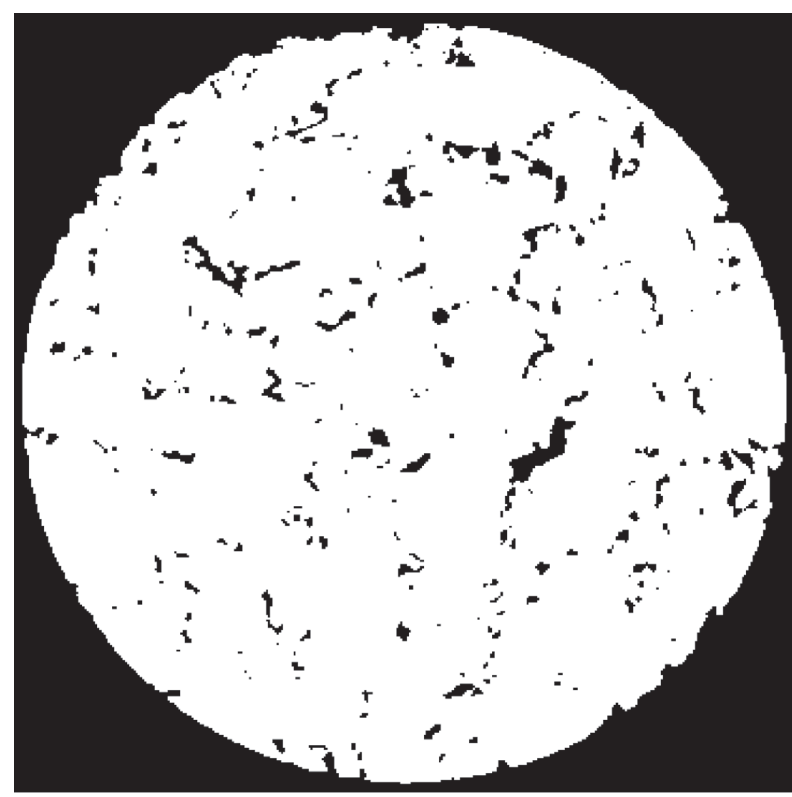

FIgURE 10: Void picture after binarization.

difference in other cases is not significant. The relationship between surface voidage and specimen height is shown in Figures 11 and 12 .

The main results are as follows:

(1) To the static compaction specimen, the voids change continuously along the depth direction of the specimen. The voidage of suspension gradation tends to increase at first then decrease and then increase again, which indicates that there are more voids in the middle and at the bottom end of the specimen. The voidage of skeleton gradation varies uniformly with depth and fluctuates up and down at
$3 \%$. The voidage of suspension gradation is larger than that of skeleton gradation.

(2) To the specimen of vibratory compaction, the surface voidage distribution of suspension gradation is still uneven, and the general trend is similar to that of static compaction. The voidage distribution of skeleton gradation is more uniform about $2 \%$, and the void is smaller than that of static compaction.

(3) Comparing the specimen compacted by the two method, the surface voidage of skeleton dense gradation is obviously lower than that of suspension dense gradation under the same method. This is because the skeleton dense gradation can be better formed into the multigrade stone-stone structure, and fine aggregate can better fill the coarse aggregate skeleton so that the inner part is more compact and even more uniform. Under the same gradation form, the surface voidage of the vibrating specimen is smaller than that of the static compaction, which is mainly due to the different effect of the two compaction methods on the specimen. The vibration method can make the mixture rearrange, but the static compaction only makes the aggregate squeeze each other through the vertical pressure, and the aggregate position will not change.

(4) Under the two compaction method, the distribution trend of the voids for the suspension gradation has no obvious difference although the voidage is reduced some what, and the void ratio of the skeleton gradation is less than that of suspension gradation, which indicates that the gradation form has more significant influence on the internal structure of the CSM. The vibration method can reduce the voidage and improve the compactness of the skeleton gradation, which also further intuitively verifies the viewpoint that the skeleton grading is more suitable for the vibration compaction method.

4.2. Distribution of Coarse Aggregate in CSM. The proportion of the coarse aggregate area of each sectorial area was calculated separately. Take the $9 \mathrm{~cm}$ section of the middle of the test piece to carry out the image analysis at the interval of $5 \mathrm{~mm}$, and each one has 18 cross-section pictures. Every cross-section picture could extract 12 coarse aggregate area ratios. The coefficient of variation of the coarse aggregate area proportion was received. Although the coarse aggregate structure of the CSM cannot be directly known by the proportion of the coarse aggregate area, it can be indirectly reflected by its variation coefficient. If the structural uniformity of the coarse aggregate of the CSM is good, the variation coefficient of the coarse aggregate area ratio will be small, otherwise the variation coefficient will be large. The variation coefficient of the coarse aggregate area ratio of the CSM under different compaction methods was shown in Figures 13 and 14. 
TABLE 3: Statistical results of the surface voidage distribution.

\begin{tabular}{lccccc}
\hline $\begin{array}{l}\text { Sample } \\
\text { ID }\end{array}$ & $\begin{array}{c}\text { Maximum surface voidage } \\
(\%)\end{array}$ & $\begin{array}{c}\text { Minimum surface voidage } \\
(\%)\end{array}$ & $\begin{array}{c}\text { Average surface voidage } \\
(\%)\end{array}$ & $\begin{array}{c}\text { Standard deviation } \\
(\%)\end{array}$ & $\begin{array}{c}\text { Coefficient of } \\
\text { variation }\end{array}$ \\
\hline XFJY & 9.04 & 1.70 & 4.75 & 1.17 & 0.25 \\
GJJY & 3.69 & 1.81 & 2.96 & 0.39 & 0.13 \\
XFZD & 5.96 & 1.84 & 3.11 & 0.79 & 0.25 \\
GJZD & 3.04 & 0.79 & 1.59 & 0.46 & 0.29 \\
\hline
\end{tabular}

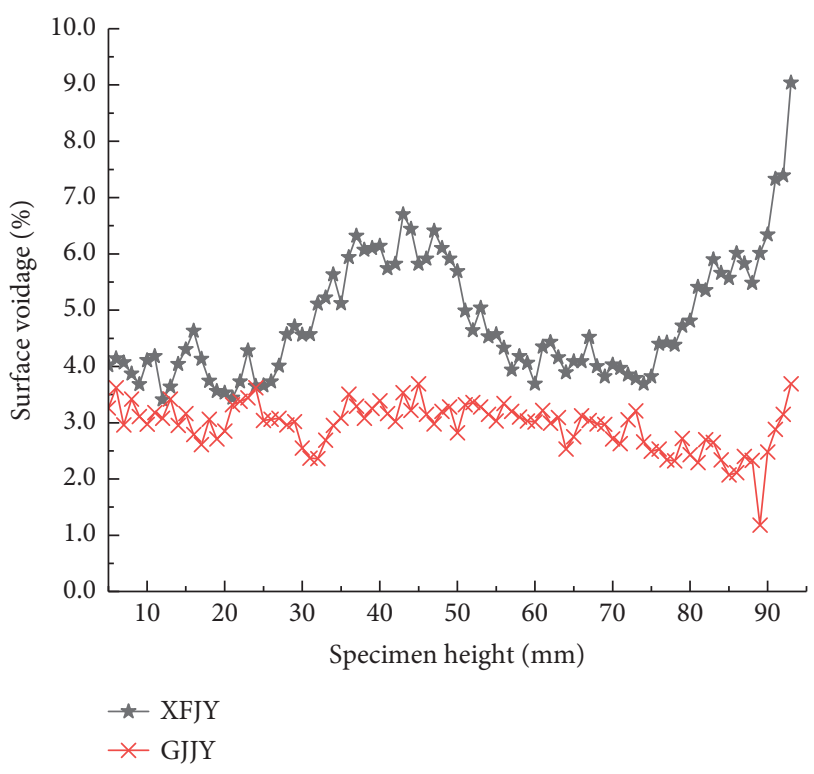

Figure 11: The void distribution characteristics of different gradation under static compaction.

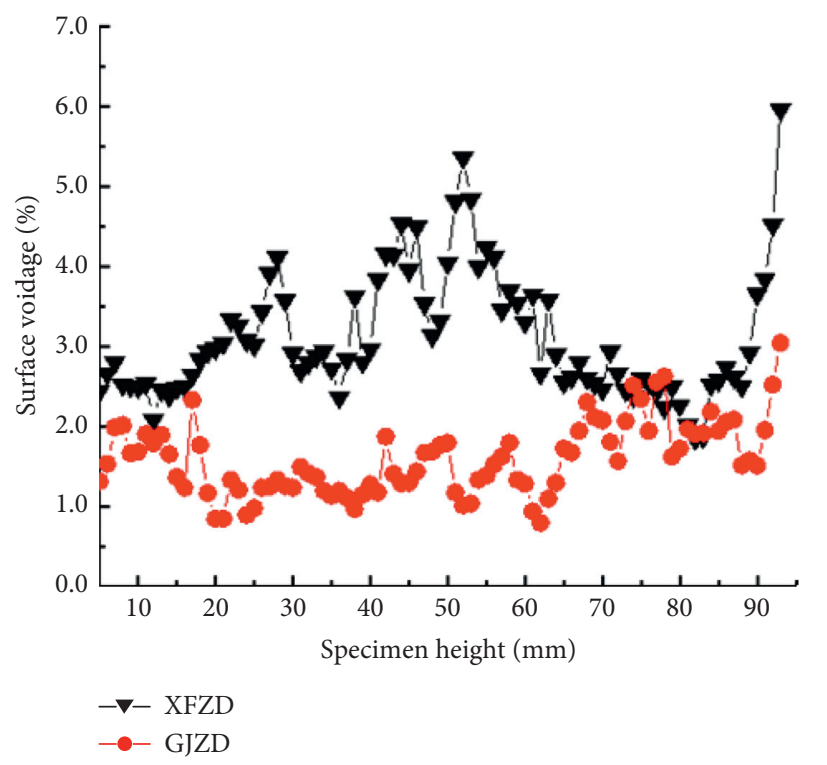

Figure 12: The void distribution characteristics of different gradation under vibration compaction.

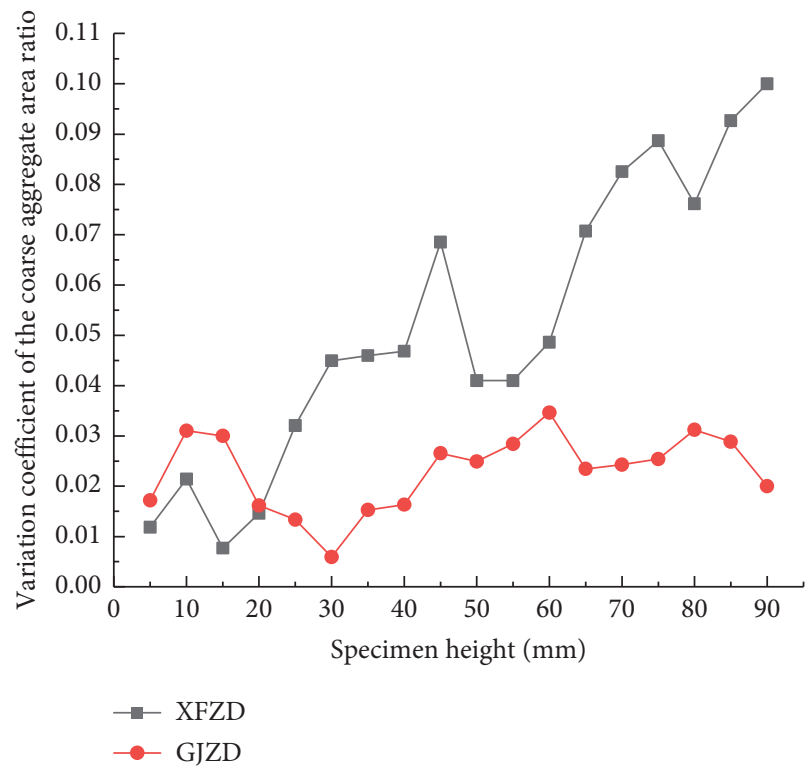

FIgURE 13: The variation coefficient of the coarse aggregate area ratio under vibration compaction.

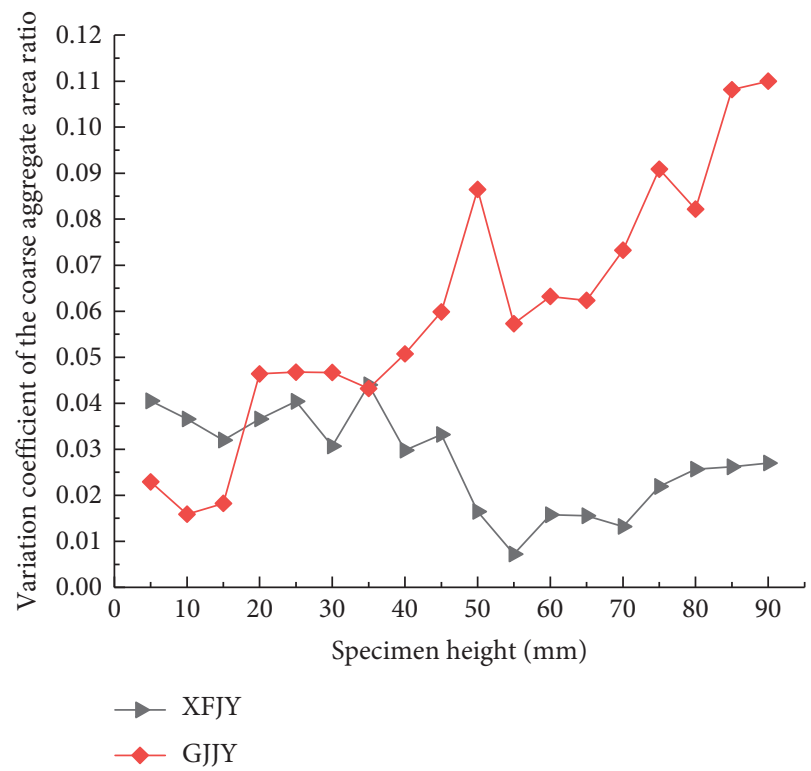

FIgURE 14: The variation coefficient of the coarse aggregate area ratio under static compaction. 
As shown in the figure above, the relationship of the variation coefficient of the area ratio of coarse aggregate with the specimen depth can be found. Under the two compaction methods, the variation coefficient of the coarse aggregate area ratio of suspended gradation is increasing, that is, along the direction of height, the structural uniformity of the suspended graded mixture is getting worse and worse. In contrast, with the increase of height, the structural uniformity of the skeleton-graded mixture shows a better trend, and it is better than that of the suspended graded mixture. It can be seen that the internal structure uniformity of CSM mainly depends on the proportion of each particle size aggregate in gradation, and the influence of compaction method is not great. The key to ensure CSM performance is to design reasonable gradation and strictly control it in construction.

The uniformity of the structure of the skeleton-graded CSM is slightly different under the two compaction methods. The structural uniformity at the upper half of the specimen height under vibration is better, while at the lower half of the specimen height the static compaction makes the inner structure more uniform, but the difference is not significant. For suspension gradation, the coarse aggregate distribution of CSM in the vibration method is more uniform than that in static compaction. On the whole, it can be seen that the vibration method can improve the coarse aggregate structural uniformity of CSM because the vibratory force may change the distribution state of the coarse and fine aggregate and lead to more uniform internal structure in the process of vibration.

\section{Summary and Conclusions}

In this paper, the internal structure characteristics of CSM were studied by industrial CT scanning. The effects of gradation and compaction methods on its internal structure void and coarse aggregate distribution uniformity were analyzed using digital image processing technology. The main conclusions are as follows:

(1) More than $80 \%$ of the voids in CSM are smaller than $1 \mathrm{~mm}^{3}$, and the number of large volume voids of skeleton dense gradation, especially $0.1 \sim 0.5 \mathrm{~mm}^{3}$, is more than that of suspended dense gradation. In general, the void volume of skeleton dense gradation is larger than that of suspension dense gradation under the two compaction methods.

(2) The surface voidage of skeleton gradation in different compaction methods is smaller than that of suspension gradation, which indicates that the gradation form has a great influence on the internal structure of CSM, and the compaction method cannot change the distribution state of the void. The distribution of voids of suspension gradation is small at both ends and large in the middle of the test specimen, and in skeleton gradation the distribution of voids is uniform generally, but the vibration method can reduce the voidage of the two kinds of CSM.
(3) The coefficient of variation of the proportion of the coarse aggregate area can be used to analyze the structural distribution of coarse aggregate in CSM. The internal structure of CSM mainly depends on the proportion of each particle size aggregate in gradation, and the influence of compaction method is not great. However, the vibration method can make the distribution of coarse aggregate structure of CSM more uniform.

(4) By analyzing the parameters of the CT scanning digital image of the CSM, it is found that the favorable gradation of the CSM and the strict control in the construction are the key to ensure its uniformity of the internal structure. On this basis, to select a reasonable compaction method can improve its degree of density and structural uniformity.

\section{Data Availability}

The data in this manuscript belongs to test results. The test data used to support the findings of this study are included within the article. There are no restrictions on data access.

\section{Conflicts of Interest}

The authors declare no conflicts of interest.

\section{Authors' Contributions}

C. L. and G. T. conceptualized the study. C. L., G. T., and Y. W. helped with the methodology; G. T. and L. Z. validated the study; C. L. and Y. W. helped with the formal analysis; Y. W. and Z. Y. helped with the investigation; C. L. and Y. W. wrote and prepare the original draft; G. T. and Y. Z. wrote and edited the review; C. L., Y. Z., and Z. Y. were responsible for project administration; C. L. and G. T were responsible for funding acquisition. All authors have read and agreed to the published version of the manuscript.

\section{Acknowledgments}

This research was funded by the Scientific and Technological Project of Transportation Department of Jilin Province (Grant no. 2018ZDGC-9-1).

\section{References}

[1] Y. Lai, Z. Wu, Y. Zhu et al., "CT analysis of freeze-thaw damage of surrounding rock in Dabanshan tunnel," Journal of Glaciology and Geocryology, vol. 22, no. 2, pp. 206-210, 2000.

[2] C. Wang, N. Guo, and Y. Tan, "Internal void characteristics of asphalt mixture with different forming methods and gradation," Journal of Jilin University (Engineering and Technology Edition), vol. 44, no. 1, pp. 74-80, 2014.

[3] N. Guo, Z. You, and Y. Tan, "Evaluation method of asphalt mixture uniformity based on CT technology (bilingual publication)," China Journal of Highway and Transport, vol. 30, no. 1, pp. 1-9, 2017.

[4] A. E. Hunter, G. D. Airey, and A. C. Collop, "Aggregate orientation and segregation in laboratory-compacted asphalt 
samples," Transportation Research Record: Journal of the Transportation Research Board, vol. 1891, no. 1, pp. 8-15, 2004.

[5] C. Hu and D. Wang, "Evaluation of dynamic compaction uniformity of asphalt mixture based on CT technology," Journal of Chang'an University: Natural Science Edition, vol. 30, no. 6, pp. 24-28, 2010.

[6] H. Azari, Effect of Aggregate Inhomogeneity on Mechanical Properties of Asphalt Mixtures, School of the University of Maryland, College Park, MD, USA, 2005.

[7] Y. Duan, X. Zhang, Li Zhi et al., "Two-dimensional and threedimensional outline characterization method of concrete aggregate based on industrial CT," China Journal of Highway and Transport, vol. 24, no. 6, pp. 9-15, 2011.

[8] S. Thyagarajan, L. Tashman, E. Masad, and F. Bayomy, "The heterogeneity and mechanical response of hot mix asphalt laboratory specimens," International Journal of Pavement Engineering, vol. 11, no. 2, pp. 107-121, 2010.

[9] S. Yang, Na Liu, H. Zhu et al., "Effect of CT Test on void fraction of Asphalt mixture," Intercommunication Science and Process, vol. 32, no. 1, pp. 7-16, 2016.

[10] X. Xiao and X. Zhang, "Study on connected void characteristics of drainage Asphalt mixture based on Industrial CT," Chinese Journal of Highway, vol. 29, no. 8, pp. 22-28, 2016.

[11] W. Wu, D. Wang, and X. Zhang, "Internal void Distribution characteristics of asphalt mixture based on Industrial CT Technology," Journal of Central South University (Natural Science Edition), vol. 43, no. 6, pp. 2343-2348, 2012.

[12] W. Tian, P. Zhang, Y. Xie et al., "Three-dimensional distribution characteristics of concrete pore structure based on CT technology in freeze-thaw environment," Journal of Chang'an University (Natural Science Edition), vol. 36, no. 3, p. 8, 2016.

[13] Y. Gao, H. Chen, C. Zou et al., "Study on meso-crack propagation of lightweight aggregate concrete based on industrial CT test," Bulletin of the Chinese Ceramic Society, vol. 33, no. 4, pp. 947-952, 2014.

[14] L. Mao, Y. Liu, and R. Xue, "Three-dimensional meso-numerical simulation and CT experimental study of concrete," Concrete, vol. 37, no. 11, pp. 7-11, 2015.

[15] W. Gong, X. Wu, and Z. Zhang, "Study on meso-damage characteristics of coal and rock based on CT scanning," Coal Science and Technology, vol. 46, no. 09, pp. 122-130, 2018.

[16] Ministry of Transport of the People's Republic of China, Technical Guidelines for Construction of Highway Roadbases (JTG/T F20-2015), Ministry of Transport of the People's Republic of China, Beijing, China, 2015, In Chinese.

[17] Ministry of Transport of the People's Republic of China, Test Methods of Materials Stabilized with Inorganic Binders for Highway Engineering (JTG E51-2009), Ministry of Transport of the People's Republic of China, Beijing, China, 2009, In Chinese.

[18] H. Liu, "Study on fine microstructure and properties of emulsified asphalt cold recycled mixture based on industrial CT," Highway Engineering, vol. 42, no. 2, pp. 319-325, 2017.

[19] W. Wu, L. Zhi, X. Zhang et al., "Using digital image processing technology to evaluate the uniformity of asphalt mixture," Journal of Jilin University (Engineering and Technology Edition), vol. 39, no. 4, pp. 921-925, 2009. 